\title{
Home Blood Pressure Self-Monitoring in Patients Treated With Anti-Angiogenic Drugs for the Detection of Arterial Hypertension
}

Damien Laneelle*, Pierre Bigot, Sophie Abadie-Lacourtoisie, Azzouzi Abdel-Rahmène, Mario Campone and Georges Leftheriotis

University hospital Angers, France

*Corresponding author: Damien Laneelle, University hospital Angers, France; Tel: 33241354617; E-mail: damien.laneelle@gmail.com

Received date: July 10, 2014; Accepted date: September 29, 2014; Published date: October 01, 2014

Copyright: (c) 2014 Laneelle D, et al. This is an open-access article distributed under the terms of the Creative Commons Attribution License, which permits unrestricted use, distribution, and reproduction in any medium, provided the original author and source are credited.

\begin{abstract}
Introduction: We compared in a prospective open-label study two different protocols of home self-measurement of arterial blood pressure (ABP) for the detection of antiangiogenic drugs (AAG)-induced arterial hypertension (AHT).

Material and methods: We performed 3 measurements every morning and evenings measurements for 3 successive days (hBP-3d) and compared them to a single daily morning measurement of BP for 7 days (hBP-7d) during 2 consecutive treatment cycles with bevacizumab or sunitinib.

Results: Among the 26 patients treated with AAG, there was a significant difference between the number of AHT episode based on hBP-3d and hBP-7d protocol $(116$ against $183, \mathrm{p}<0.0005)$. AHT did not correlated with tumoral progression/stabilization and no significant predictability could be established using the 2 protocols.
\end{abstract}

Conclusion: Detection of AHT episod in patients treated with AAG was linked to the BP monitoring protocol and should be specifically designed for cancer patients treated with AAG.

Keywords: Antiangiognenic; Induced hypertension; Cancer; Home blood pressure monitoring

\section{Introduction}

Arterial hypertension (AHT) is a common side effect observed with antiangiogenic (AAG) treatments with the percentage of cases ranging from $11 \%$ to $43 \%[1,2]$, depending on the molecule, the dose and the definition of hypertension, this produce an increase of cardiovascular risk compared to the general population [3]. The mechanism of increased arterial blood pressure (BP) with treatment using AAG drugs is not fully understood, this mechanism is multifactorial, and includes endothelial dysfunction, capillary rarefaction and dysautonomia [4]. Vascular endothelial growth factor (VEGF) signaling represents a critical step in the process of angiogenesis [5], and agents targeting VEGF are being extensively investigated as anticancer therapy [6]. VEGF not only drives angiogenesis [7] but also serves as a survival factor for endothelial cells and contributes to the promotion of an abnormal phenotype of blood vessels in tumors [8]. Whatever their initial level of blood pressure, every patient receiving antiangiogenic treatment evidenced rapid and large increases in blood pressure; in most cases, the blood pressure values did not reach the levels characterizing clinical hypertension [9]. Mourad et al. have shown that mean BP was increased after 6 months of AAG therapy compared with baseline, from $129 \pm 13 / 75 \pm 7 \mathrm{mmHg}$ to $145 \pm 17 / 82 \pm$ $7 \mathrm{mmHg}$ for systolic BP and diastolic BP respectively $(\mathrm{p}<0.0001)$ [10]. For patients treated with AAG, home blood pressure monitoring (HBPM) allows a better BP control, fewer complications and improved overall survival $[4,11]$ and the role of hypertension in determining the risk of coronary artery disease is well known [12]. Several studies have suggested that early blood pressure rise was associated with better antitumoral efficacy and improved prognosis, making this commonly observed effect a promising marker of efficacy [13]. However, the best method for monitoring BP (i.e. readings per day and number of days measured) during AAG treatment remains to be validated in order to improve the detection and control of AHT specifically induced by AAG. Recommendations about HBPM have been forwarded in 2008 [14]. They suggest 3 consecutive measurements in the morning and evening for 3 days, resulting in the diagnosis of AHT if BP values (averaged over 18 measurements) exceeded 135/85 mmHg. The National Cancer Institute (NCI) proposed another protocol with a single daily measurement and different diagnosis thresholds. Definitions of AHT also differ and the last version of the Common Terminology Criteria for Adverse Events published by the National Cancer Institute (NCI-CTCAE) [15] defined AHT by a transient $(<24$ hrs) increase in diastolic $(>20 \mathrm{mmHg})$ or systole/diastole BP $(>150 / 100 \mathrm{mmHg})$. Finally, the severity of AHT, and thus its management, is graded in 6 levels, from low (i.e. grade 0 ) to lifethreatening state with hypertensive crisis (i.e. grade 5).

The purpose of this prospective open-label study was to compare two different HBPM protocols differing by the frequency of measurements and the threshold of AHT in patients treated with AAG.

\section{Material and Methods}

\section{Population}

All patients were recruited from the University Hospital of Angers and the oncology department of the Institut de Cancérologie de l'Ouest (Angers-France) for the treatment of a solid tumor (kidney, breast or GI tract). Antiangiogenic treatments prescribed in the study were bevacizumab (BEVACIZUMAB, Roche, France), a humanized 
Citation: Laneelle D, Bigot P, Abadie-Lacourtoisie S, Abdel-Rahmène A, Campone M, et al. (2014) Home Blood Pressure Self-Monitoring in Patients Treated With Anti-Angiogenic Drugs for the Detection of Arterial Hypertension. J Hypertens 3: 179. doi: 10.4172/2167-1095.1000179

Page 2 of 5

anti-body against VEGF or sunitinib (SUNITINIB, Pfizer, USA), an oral tyrosine-kinase inhibitor. Both drugs were administered at usual doses, alone or in combination with the other conventional therapys (chemotherapy and radiotherapy) as a first or subsequent line of treatment. Bevacizumab was administered intravenously $(10 \mathrm{mg} / \mathrm{kg})$ the first day of the cycle (D1) and 15 days later in the cycle (D15). Sunitinib was taken orally with doses ranging from 15 to $50 \mathrm{mg}$ daily for 4 weeks followed by a 2 -week recovery interval. In both groups, all patients were included before they started a new treatment cycle, whatever the number of previous treatment cycles. Patients with an expected survival rate $>1$ year, in good clinical condition and able to perform the home self-measurements participated in the study and gave their informed consent. The study was approved by our local ethics committee.

\section{Material}

All hBP readings were performed using a validated ambulatory automated oscillometric device (OMRON M6, Kyoto, Japan) equipped with an appropriate cuff size, memory for recording results [16], and is validated for BP self-monitoring [17].

\section{Home BP measurement protocols}

The measurement protocols for HBPM are showd in Figure 1.

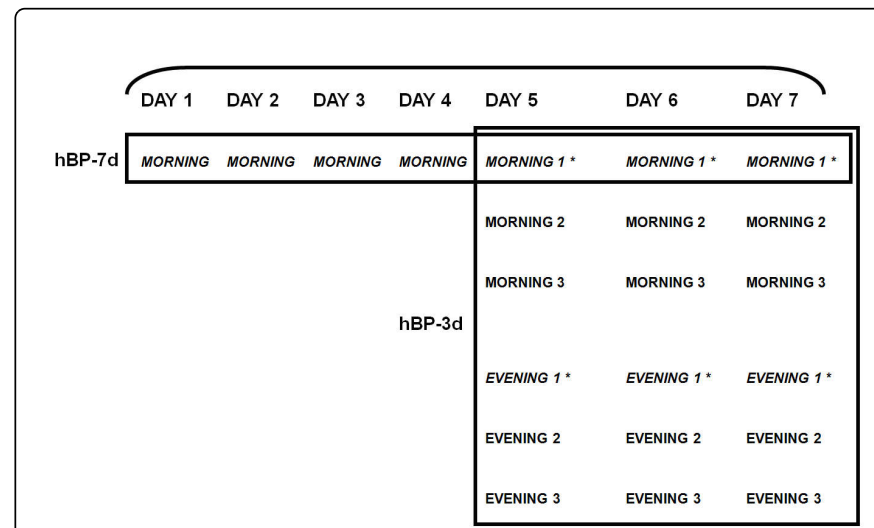

Figure 1: Measurement protocols for HBPM

All patients were asked to do early morning BP for a week. For the last 3 days of the week, in addition to the one early morning measurement, the patients were also required to do more morning as well as evening BP measurements, according to our guidelines for HBPM [18]. For the morning, 2 additional BP measurements needed to be done following the initial morning measurement. In the evenings of the same 3 days, 3 BP measurements needed to be taken. These measurements were done for 3 consecutive days during the last 3 days of the said week. We realized arithmetic averages of BP measurements over 7 or 3 days, patient by patient and week by week. When SBP week average and/or DBP week average reached the threshold, we diagnosed an AHT event for hBP-3d protocol, hBP-7 protocol, or both. For the $\mathrm{hBP}-3 \mathrm{~d}$ protocol the first morning and evening measurements of each day were discarded and the remaining two readings (i.e. 12 readings) were averaged for the 3 days. The threshold for AHT as defined in the NCI-CTCAE v. 4 was applied for the hBP-7d protocol [7] (i.e. systolic $\mathrm{BP}$ value $\geq 140-159 \mathrm{mmHg}$ and/or diastolic 90-99 $\mathrm{mmHg}$ ). The threshold for AHT validated for the HBPM (ie. systolic $\geq 135 \mathrm{mmHg}$ and/or diastolic $\geq 85 \mathrm{mmHg}$ ) [19] was applied to the readings from the $\mathrm{hBP}-3 \mathrm{~d}$ protocol. In all groups and whatever the treatment, $\mathrm{hBP}$ measurement was performed before AAG treatment was started (ie. Baseline BP), achieve in one day. All hBP measurements were performed each week of treatment for 4 weeks and for 2 consecutive treatment cycles. Patients treated with sunitinib performed the HBPM every week for 4 weeks. During the off-treatment/recovery phase of 2 weeks in those receiving sunitinib, BP measurements were recorded and analyzed separately. Each patient was asked to self-report adverse effects for each treatment cycle, and if medical advice or any intervention was required such as antihypertensive medications and/or drugs dose schedule modifications. The day of inclusion, biometric data, oncologic status and treatments were recorded.

\section{Statistical analysis}

All quantitative variables are given as mean $( \pm \mathrm{SD})$ and qualitative variables are presented as percentage. Statistically significant differences between paired qualitative variables were determined by McNemar's and Fisher's tests for unpaired variables. The difference between the 2 measurement protocols to detect AHT was determined by McNemar's test. For all statistics, a $p$ value $<0.05$ was considered significant.

\section{Results}

\section{Population}

Patients and drugs characteristics are described in Table 1.

\begin{tabular}{|l|l|l|}
\hline \multicolumn{1}{|c|}{ Variable } & No. & $\%$ \\
\hline Gender & & \\
\hline Male & 16 & $62 \%$ \\
\hline Female & 10 & $38 \%$ \\
\hline Age, yr & & \\
\hline Mean & 61 & \\
\hline Range & $49-77$ & \\
\hline Tumor type & & \\
\hline Kidney & 15 & $58 \%$ \\
\hline Breast & 3 & $12 \%$ \\
\hline Gastrointestinal tract & 8 & $30 \%$ \\
\hline Antiangiogenic treatment & 12 & $50 \%$ \\
\hline bevacizumab & 13 & $54 \%$ \\
\hline sunitinib & 13 & \\
\hline HTN prior to treatment & & $50 \%$ \\
\hline yes & & \\
\hline No & & \\
\hline
\end{tabular}

Table 1: Patients and drugs characteristics $(n=26)$ 
Citation: Laneelle D, Bigot $P$, Abadie-Lacourtoisie S, Abdel-Rahmène A, Campone M, et al. (2014) Home Blood Pressure Self-Monitoring in Patients Treated With Anti-Angiogenic Drugs for the Detection of Arterial Hypertension. J Hypertens 3: 179. doi: 10.4172/2167-1095.1000179

Page 3 of 5

Twelve patients (46\%) were treated by 1 or more anti-hypertensive drugs prior to entering the study and no one was a smoker. One serious adverse event occurred (thrombocytemia with Sunitinib), but no cardiovascular event and no patient died during the study. Only 15 patients (57\%) claimed to do all measurements but all completed the study.

\section{Arterial hypertension during AAG treatment}

The difference between the 2 measurement protocols to detect AHT for both protocols is summarized in Table 2 .

\begin{tabular}{|l|l|l|l|l|}
\hline & & \multicolumn{2}{|l|}{ hBP-3d } & \\
\hline & & AHT - (n) & AHT + (n) & total \\
\hline \multirow{3}{*}{ hBP-7d } & AHT - (n) & 145 & 11 & 156 \\
\cline { 2 - 5 } & AHT + (n) & 38 & 78 & $116^{*}$ \\
\hline & total & 183 & $89^{*}$ & 272 \\
\hline
\end{tabular}

Table 2: Difference between the 2 measurement protocols to detect AHT

Mac Nemar's test, AHT: Arterial Hypertension, *: Statistical difference

A statistical difference $(\mathrm{p}<0.0005)$ was observed between the "hBP-3d" and "hBP-7d" protocols ( $\mathrm{n}=272$ measures) with a Kappa value of 0.62 . The arterial blood pressure was significantly higher with sunitinib (SBP: $139 \pm 16 \mathrm{mmHg}$, DBP: $78 \pm 14 \mathrm{mmHg}$ ) than with bevacizumab (SBP: $127 \pm 13 \mathrm{mmHg}, \mathrm{DBP}: 77 \pm 8 \mathrm{mmHg}, \mathrm{p}<0.05$ ), compared to pre-treatment arterial blood pressure with sunitinib (SBP: $132 \pm 17 \mathrm{mmHg}$, DBP: $76 \pm 10 \mathrm{mmHg}$ ) and bevacizumab (SBP: $129 \pm 18 \mathrm{mmHg}$, DBP: $78 \pm 11 \mathrm{mmHg}$ ). The SBP and DBP values with both drugs are listed in figure 2 .

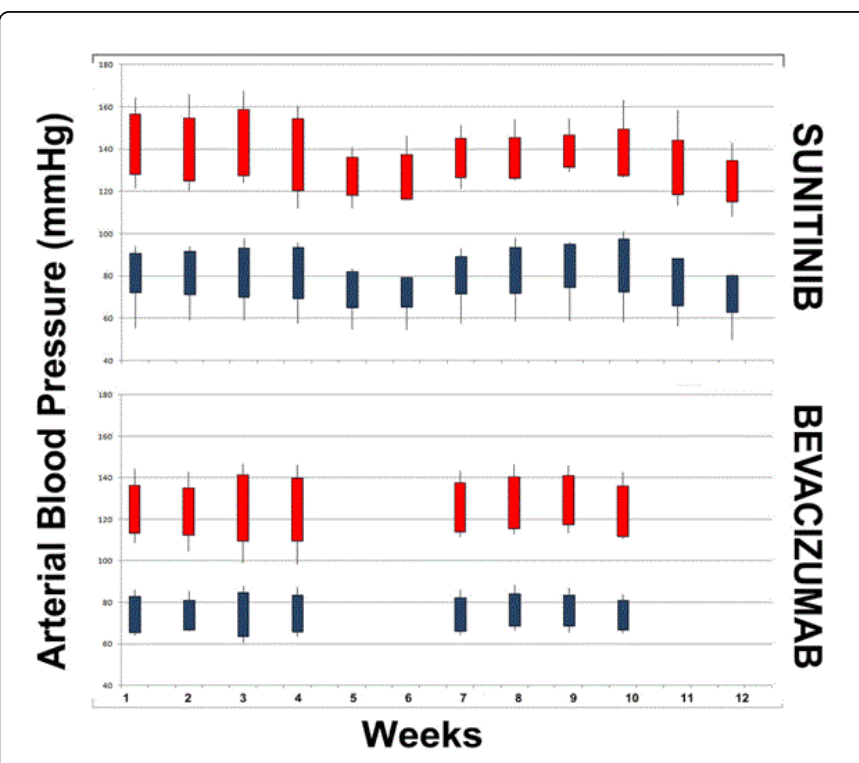

Figure 2: Systolic and Diastolic blood pressure with both drugs

According to the NCI CTCAE v4.0 threshold, pre-hypertension (grade 1) was observed in $11 \%$ of patients. The hypertensive grades 2 and 3 were more frequent with sunitinib (84\%) than bevacizumab (36\%) and no grades 4 or 5 were observed during the study or lifethreatening complications (i.e. malignant AHT and posterior reversible leucoencephalia). Independently from the reading protocols and the AAG drugs $61 \%$ of the BP values reached the threshold for AHT. Patients treated with sunitinib performed the HBPM every week for 4 weeks. No statistical difference was observed between BP baseline and the off-treatment/recovery phase of 2 weeks in those receiving sunitinib.

\section{Relationship between arterial hypertension, survival and tumoral progression}

The relationship between progression free and overall survival and hypertensive grades (NCI CTC $v 4.0$ definition) is shown in table 3 for both AAG drugs. There was no significant relationship between the presence/absence of AHT detected either with NCI-CTCAE v4.0 criteria or using the JNC7 definition $(>135 / 85 \mathrm{mmHg}$ ) and the tumoral progression/survival (Table 3 ). The sensitivity and specificity to detect a tumoral progression or death using hBP-3d was $53 \%$ and $46 \%$ respectively and $61 \%$ and $38 \%$ for hBP-7d (no significant difference). The positive and negative predictive values for hBP-3d were $53 \%$ and $46 \%$, respectively, and $50 \%$ and $50 \%$ for the hBP-7d protocol (no significant difference).

\begin{tabular}{|l|l|l|l|l|l|l|}
\hline \multirow{2}{*}{} & \multicolumn{5}{|c|}{ NCI-CTCAE v4.0 hypertensive grades } & \multirow{2}{*}{ Overall } \\
\cline { 2 - 7 } & grade 0 & $\begin{array}{l}\text { grade } \\
\mathbf{1}\end{array}$ & grade 2 & grade 3 & grade 4 & \\
\hline $\begin{array}{l}\text { Survival } \\
\text { without } \\
\text { progression }\end{array}$ & 4 & 1 & 5 & 3 & 0 & 13 \\
\hline $\begin{array}{l}\text { Progression } \\
\text { or death }\end{array}$ & 3 & 2 & 5 & 3 & 0 & 13 \\
\hline Overall & 7 & 3 & 10 & 6 & 0 & 26 \\
\hline
\end{tabular}

\begin{tabular}{|l|l|l|l|}
\hline \multirow{2}{*}{} & \multicolumn{2}{|c|}{ JNC7 ambulatory hypertension } & Overall \\
\cline { 2 - 3 } & $<135 / 85 \mathrm{mmHg}$ & $>135 / 85 \mathbf{m m H g}$ & \\
\hline $\begin{array}{l}\text { Survival without } \\
\text { progression }\end{array}$ & 7 & 8 & 15 \\
\hline Progression or death & 6 & 7 & 11 \\
\hline Overall & 11 & 15 & 26 \\
\hline
\end{tabular}

Table 3: Survival and progression rate of patients according to the arterial hypertension NCI-CTCAE $\mathrm{v} 4.0$ grades and ambulatory definition.

\section{Discussion}

This prospective open-label study compared 2 different protocols of HBPM for the detection of AHT in patients treated with AAG. The protocols differed by the frequency of readings for $\mathrm{BP}$ and the threshold to detect AHT. In our study, $61 \%$ of the BP values reached the threshold for AHT independently from the reading protocols and the AAG drugs. Although we observed a statistical difference between the two measurement protocols, the study design cannot determine which protocol was better at detecting AHT in the absence of a 
reference method. However with a Kappa value of 0.62 it might seem, therefore, that this difference does not prevent a fairly good correlation between the two protocols. As a result, the least restrictive protocol should be preferred and hBP-7d, with only 7 measurements per week (compared to 18 for hBP-3d), is probably the least restrictive.

Our data also showed that AHT did not correlated with tumoral progression/stabilization and no significant predictability could be established using the 2 protocols.

According to the European JNC7 recommendations, and as opposed to the office blood pressure measurement, the use of ambulatory or home self-measurements are well-adapted for the detection of AHT allowing closer monitoring and reducing false detection of AHT such as the "white coat" effect [20]. Contrary to the casual office measurement, the use of a $24 \mathrm{~h}$-ambulatory BP Holter [21] or a home ambulatory BP device are more suited for tracking rapid changes in BP induced by AAG treatments [5] although these methods represent a supplemental constraint for these patients. The detection, and thus the management, of AHT are also dependent upon the threshold retained for AHT. Threshold values for AHT are lower with hBP (i.e. $135 / 85 \mathrm{mmHg}$ ) than the office BP (i.e $140 / 90 \mathrm{mmHg}$ ) [4]. Although the use of hBP is recommended to diagnose AHT, both the measurement protocol and thresholds values for AHT could greatly influence the detection and management of AHT during AAG therapy.

According to the literature [22-25], patients treated with AAG showed an increase in the systemic BP which was significant with sunitinib compared to bevacizumab. Consistent and rapid changes in the ambulatory BP are usually observed within the first 2 weeks [13] of treatment leading to more intensive monitoring during this period. Accordingly, daily monitoring has been recommended for sunitinib and sorafenib [26], mostly during the first cycle [16]. It has also been demonstrated that an earlier antihypertensive therapy is beneficial to achieve uninterrupted, full dose therapy in most patients [13]. In this view, the potential importance of physical exercise should be considered in further studies [27]. Furthermore, inappropriate detection of AHT caused by interfering factors could limit the efficacy of AAG therapys. Additionally, the choice for systolic and diastolic threshold values for AHT has been legitimized by population-based studies.

In the general population, it has been shown that increasing the number of readings reduces the variability and thus improved the diagnosis of essential hypertension [28] and steady BP readings are generally obtained with day-to-day measurements [29]. In the case of AAG, the fact that AHT could develop rapidly [5] strongly argues for daily measurements. Therefore, considering our data, it is likely that the ideal protocol would be a mixture of the 2 protocols with, for example, daily measurements twice per day.

Another important characteristic in the BP monitoring of our AAG-treated patients was represented by the change from the pretreatment values. In our study, we observed limited but significant changes of SBP, but not DBP, between bevacizumab and sunitinib and the increase was more apparent for DBP suggesting an increase in the peripheral resistance. By contrast, bevacizumab showed insignificant changes in both SBP and DBP rather suggesting a change in the arterial compliance. Indeed, the large variability in the BP changes indicates a broad range of individual systemic response to AAG that should be further evaluated to determine its potential interest in the monitoring of AAG treatment.
A potential clinical interest of ahBP protocol to monitor the hypertensive effect during AAG could be evaluated by the survival and rate of tumoral progression criteria. In contrast to previous studies showing that a higher rate of survival without progression is linked to the presence of AHT [5,30-31] we could not demonstrate a relationship between the rate of tumoral progression and death and the presence/absence of AHT. This study was not designed to evaluate the blood pressure (BP) as a prognostic marker of TKI response. Indeed we included different tumour types and different drugs to assess the correlation between two different blood pressure measurement protocols. For example, the study of Osterlund et al. included 100 patients with colorectal cancer treated by bevacizumab in order to validate BP as a surrogate marker of treatment response [32].

Contrary to other studies, BP was assessed by self-measurement at home using a validated automatic device. Indeed, when compared to $24 \mathrm{~h}$ ambulatory BP monitoring, the number of readings is comparably low but compatible with a sustainable constraint for the patient, at a lower expense and with comparable results between both techniques. Furthermore, most of the patients expressed a preference for the hBP-7d over the hBP-3d protocol.

\section{Study limitations}

A main limitation was the small sample of patients and the heterogeneity of treatments and their cycles. The fact that sunitinib induced more AHT during the first cycle than during the remaining cycles certainly added to the variability of the data. However, this variability also reflects a limitation to transfer the data issued from large general population studies to an individual prognostic capable of BP monitoring to predict the response to treatments. Another limitation is the definition of baseline blood pressure. Pre-treatment hBP measurement was performed in one day; it will be interesting to define a pre-treatment value of at least one week.

\section{Conclusions}

Home self-measured BP represents an attractive method for the hemodynamic monitoring of patients treated with AAG. Both protocols compared in this study differ by the detection threshold and frequency of measurements. They are regularly used in the oncologic field (7d-hBP proposed by the National Cancer Institute) and the cardiological field (3d-hBP). The hBP-7d protocol appears to be equivalent to the hBP-3d protocol in terms of detection of AAGinduced AHT. As a result, the least constraining protocol (hBP-7d) should be preferred. Indeed, further studies are still needed to determine the best adapted BP monitoring and thresholds for the individual management of AHT induced by AAG therapys and to redefine its role in the predictability of AAG efficacy.

\section{Acknowledgement}

The study was funded by "ligue contre le cancer 49 ".

\section{References}

1. Hurwitz H, Fehrenbacher L, Novotny W, Cartwright T, Hainsworth J, et al. (2004) Bevacizumab plus irinotecan, fluorouracil, and leucovorin for metastatic colorectal cancer. N Engl J Med 350: 2335-2342.

2. Yang JC, Haworth L, Sherry RM, Hwu P, Schwartzentruber DJ, et al. (2003) A randomized trial of bevacizumab, an anti-vascular endothelial growth factor antibody, for metastatic renal cancer. N Engl J Med 349: 427-434. 
Citation: Laneelle D, Bigot P, Abadie-Lacourtoisie S, Abdel-Rahmène A, Campone M, et al. (2014) Home Blood Pressure Self-Monitoring in Patients Treated With Anti-Angiogenic Drugs for the Detection of Arterial Hypertension. J Hypertens 3: 179. doi: 10.4172/2167-1095.1000179

Page 5 of 5

3. Santulli G (2013) Epidemiology of Cardiovascular Disease in the 21st Century: Updated Numbers and Updated Facts. Journal of Cardiovascular Disease 1: 1 .

4. Mourad JJ, Levy BI (2011) Mechanisms of antiangiogenic-induced arterial hypertension. Curr Hypertens Rep 13: 289-293.

5. Ferrara N (2004) Vascular endothelial growth factor: basic science and clinical progress. Endocr Rev 25: 581-611.

6. Santulli G, Basilicata MF, De Simone M, Del Giudice C, Anastasio A, et al. (2011) Evaluation of the anti-angiogenic properties of the new selective $\hat{\mathrm{I}} \pm \mathrm{VI}^{2} 3$ integrin antagonist RGDechiHCit. J Transl Med 9: 7.

7. Santulli G, Ciccarelli M, Palumbo G, Campanile A, Galasso G, et al. (2009) In vivo properties of the proangiogenic peptide QK. J Transl Med 7: 41 .

8. Inai T, Mancuso M, Hashizume H, Baffert F, Haskell A, et al. (2004) Inhibition of vascular endothelial growth factor (VEGF) signaling in cancer causes loss of endothelial fenestrations, regression of tumor vessels, and appearance of basement membrane ghosts. Am J Pathol 165: 35-52.

9. Azizi M, Chedid A, Oudard S (2008) Home blood-pressure monitoring in patients receiving sunitinib. N Engl J Med 358: 95-97.

10. Mourad JJ, des Guetz G, Debbabi H, Levy BI (2008) Blood pressure rise following angiogenesis inhibition by bevacizumab. A crucial role for microcirculation. Ann Oncol 19: 927-934.

11. Bamias A, Lainakis G, Manios E (2009) Could rigorous diagnosis and management of hypertension reduce cardiac events in patients with renal cell carcinoma treated with tyrosine kinase inhibitors? J Clin Oncol. 27: 2567-2569

12. Santulli G (2012) Coronary heart disease risk factors and mortality. JAMA 307:1137.

13. Scartozzi M, Galizia E, Chiorrini S, Giampieri R, Berardi R, et al. (2009) Arterial hypertension correlates with clinical outcome in colorectal cancer patients treated with first-line bevacizumab. Ann Oncol 20: 227-230.

14. Halimi JM, Azizi M, Bobrie G, Bouché O, Deray G, et al. (2008) [Vascular and renal effects of anti-angiogenic therapy]. Nephrol Ther 4 602-615.
15. National Cancer Institute (2009) Common Terminology Criteria for Adverse Events v4.0. NCI, NIH, DHHS. NIH publication \# 09-7473.

16. Celis H, Den Hond E, Staessen JA (2005) Self-measurement of blood pressure at home in the management of hypertension. Clin Med Res 3: $19-26$.

17. http://ansm.sante.fr/content/download/20191/245125/version/12/file/ Liste-autotensiometres-humeraux_Aout2012.pdf

18. http://www.has-sante.fr/portail/upload/docs/application/pdf/2011-09/ hta_2005_-_recommandations.pdf

19. The Task Force for the management of arterial hypertension of the European Society of Hypertension (ESH) and of the European Society of Cardiology (ESC) 2013 ESH/ESC Guidelines for the management of arterial hypertension. European Heart Journal 34: 2159-2219.

20. Celis H, Den Hond E, Staessen JA (2005) Self-measurement of blood pressure at home in the management of hypertension. Clin Med Res 3: 19-26.

21. Bamias A, Manios E, Karadimou A, Michas F, Lainakis G, et al. (2011) The use of 24-h ambulatory blood pressure monitoring (BPM) during the first cycle of sunitinib improves the diagnostic accuracy and management of hypertension in patients with advanced renal cancer. European Journal of Cancer 47:1660-1668.

22. Kappers MH, van Esch JH, Sleijfer S, Danser AH, van den Meiracker AH (2009) Cardiovascular and renal toxicity during angiogenesis inhibition: clinical and mechanistic aspects. J Hypertens 27: 2297-2309.

23. Mir O, Ropert S, Alexandre J, Goldwasser F (2009) Hypertension as a surrogate marker for the activity of anti-VEGF agents. Ann Oncol 20: 967-970.

24. Bamias A, Lainakis G, Manios E, Koroboki E, Gyftaki R, et al. (2009) Diagnosis and management of hypertension in advanced renal cell carcinoma: prospective evaluation of an algorithm in patients treated with sunitinib. J Chemother 21: 347-350. 